\title{
In Vivo PET Imaging of Cardiac Presynaptic Sympathoneuronal Mechanisms in the Rat
}

\author{
Dnyanesh N. Tipre, James J. Fox, Daniel P. Holt, Gilbert Green, Jianhua Yu, Martin Pomper, Robert F. Dannals, \\ and Frank M. Bengel \\ Division of Nuclear Medicine, Russell H. Morgan Department of Radiology and Radiological Science, Johns Hopkins University, \\ Baltimore, Maryland
}

\begin{abstract}
The sympathetic nervous system of the heart plays a key role in the pathophysiology of various cardiac diseases. Small-animal models are valuable for obtaining further insight into mechanisms of cardiac disease and therapy. To determine the translational potential of cardiac neuronal imaging from rodents to humans, we characterized the rat sympathetic nervous system using 3 radiotracers that reflect different subcellular mecha-

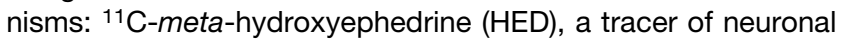
transport showing stable uptake and no washout in healthy humans; ${ }^{11} \mathrm{C}$-phenylephrine (PHEN), a tracer of vesicular leakage and intraneuronal metabolic degradation with initial uptake and subsequent washout in humans; and ${ }^{11} \mathrm{C}$-epinephrine (EPI), a tracer of vesicular storage with stable uptake and no washout in humans. Methods: We used a small-animal PET system to study healthy male Wistar rats at baseline, after desipramine (DMI) pretreatment (DMI block), and with DMI injection 15 min after tracer delivery (DMI chase). The rats were kept under general isoflurane anesthesia while dynamic emission scans of the heart were recorded for $60 \mathrm{~min}$ after radiotracer injection. A myocardial retention index was determined by normalizing uptake at $40 \mathrm{~min}$ to the integral under the arterial input curve. Washout rates were determined by monoexponential fitting of myocardial time-activity curves. Results: At baseline, HED showed high myocardial uptake and sustained retention, EPI showed moderate uptake and significant biphasic washout, and PHEN showed moderate uptake and monoexponential washout. The average ( \pm SD) left ventricular retention index for HED, PHEN, and EPI was $7.38 \% \pm$ $0.82 \% / \mathrm{min}, 3.43 \% \pm 0.45 \% / \mathrm{min}$, and $4.24 \% \pm 0.59 \% / \mathrm{min}$, respectively; the washout rate for HED, PHEN, and EPI was $0.13 \% \pm 0.23 \% / \mathrm{min}, 1.13 \% \pm 0.35 \% / \mathrm{min}$, and $0.50 \% \pm$ $0.24 \% / \mathrm{min}$, respectively. The DMI chase resulted in increased washout only for HED. DMI block decreased myocardial uptake of all tracers by less than $90 \%$. Conclusion: Kinetic profiles of HED in the rat myocardium were similar to those of HED in humans, suggesting comparable neuronal transport density. Unlike in humans, however, significant washout of EPI and faster washout of PHEN were encountered, consistent with high intraneuronal metabolic activity, high catecholamine turnover, and reduced vesicular storage. This evidence of increased neuronal activity in rodents has implications for translational studies of cardiac neuronal biology in humans.
\end{abstract}

Received Dec. 22, 2007; revision accepted Mar. 19, 2008.

For correspondence or reprints contact: Dnyanesh N. Tipre, Russell $\mathrm{H}$. Morgan Department of Radiology and Radiological Science, Johns Hopkins University, 601 N. Caroline St., JHOC 3225, Baltimore, MD 21287-0842.

E-mail: dnyanesht@yahoo.com

COPYRIGHT @ 2008 by the Society of Nuclear Medicine, Inc.
Key Words: ${ }^{11} \mathrm{C}-m e t a-h y d r o x y e p h e d r i n e ; ~{ }^{11} \mathrm{C}$-phenylephrine; ${ }^{11} \mathrm{C}$-epinephrine; autonomic nervous system; rat heart; smallanimal PET

J Nucl Med 2008; 49:1189-1195

DOI: 10.2967/jnumed.107.050252

$\mathbf{T}$ he sympathetic and parasympathetic cardiac neuronal networks play an important role in the regulation of cardiovascular performance. Although parasympathetic innervation exerts inhibitory effects via activation of muscarinic receptors, sympathetic innervation exerts a cardiac stimulatory effect (increased force of contraction and acceleration of heart rate and conduction) via activation of adrenergic receptors by norepinephrine $(1,2)$. Impairments of the functional integrity of sympathetic nerve terminals in the heart have been shown to be a key component of diseases such as congestive heart failure, dysrhythmia, ischemia, dysautonomia, Parkinson's, and diabetes (3-8). PET allows for the noninvasive assessment of cardiac sympathetic neuronal integrity using several radiolabeled catecholamine analogs (9).

${ }^{11} \mathrm{C}$-meta-hydroxyephedrine (HED), a metabolically resistant catecholamine analog, is recognized as a tracer of neuronal transport (10). ${ }^{11} \mathrm{C}$-phenylephrine (PHEN) is another catecholamine analog that, in contrast to HED, is a substrate to monoamine oxidase (MAO) and thus metabolized intraneuronally. It is considered a marker of leakage from intraneuronal storage vesicles and subsequent metabolism (11). ${ }^{11} \mathrm{C}$-epinephrine (EPI) is a true catecholamine that, in contrast to HED and PHEN, is efficiently stored in intraneuronal vesicles, where it is protected from metabolic degradation under normal conditions. It is thus considered to be a marker of neuronal uptake and vesicular storage (12). Figure 1 illustrates the neuronal pathways tracked by these 3 tracers.

All 3 radiotracers have been used in humans to assess the sympathetic nervous system. HED and EPI showed stable uptake and no washout, whereas PHEN showed uptake and subsequent washout in healthy volunteers (13-15). The kinetic profiles of these radiotracers were also studied in 


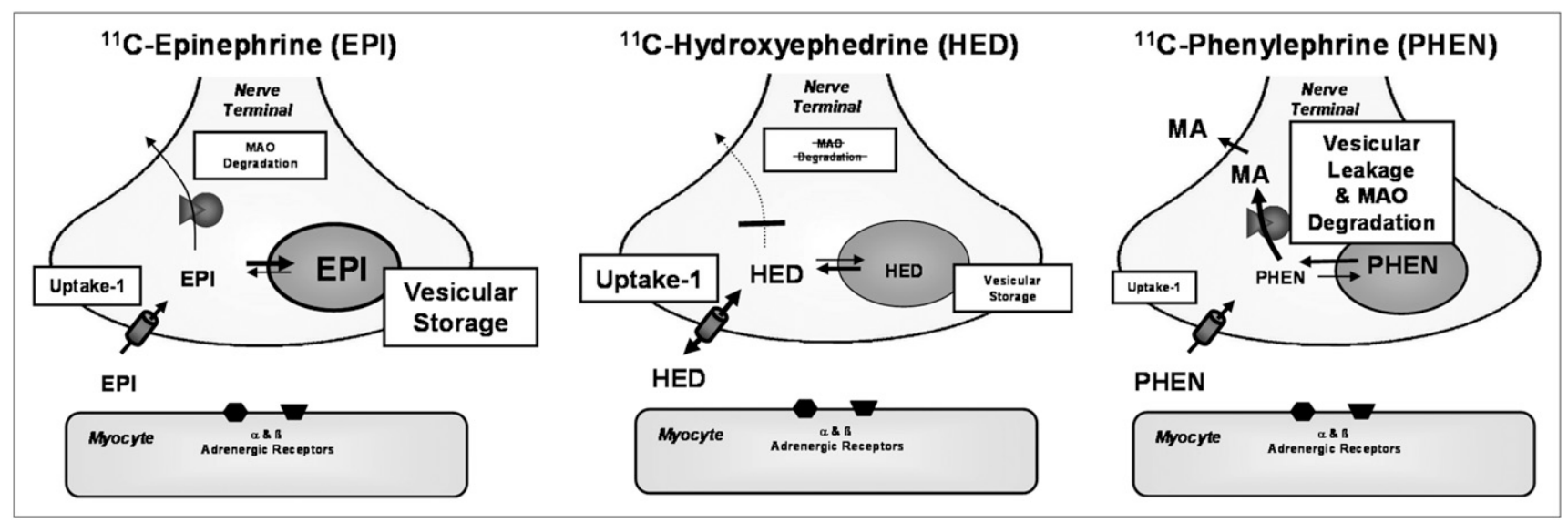

FIGURE 1. Schematic display of neuronal pathways tracked by 3 different sympathoneuronal PET tracers.

vitro in the isolated perfused rat heart model. However, tracer kinetics in the living rat may not be similar to isolated heart perfusion studies because of systemic effects and central-peripheral nervous system interaction.

The rat is a well-established animal model for cardiovascular research and drug discovery. Although the use of a single tracer provides a snapshot of the integrity of innervation, we speculated that the combination of multiple tracers with different kinetic properties would provide further insights into sympathetic nerve terminal biology. Thus, to determine the translational potential of in vivo cardiac neuronal imaging from rats to humans and vice versa, we sought to characterize the rat sympathetic nervous system using HED, PHEN, and EPI together with small-animal PET methodology.

\section{MATERIALS AND METHODS}

\section{Radiopharmaceuticals}

HED, PHEN, and EPI were synthesized as previously described $(11,16,17)$. The specific radioactivities at the end of synthesis were in the range of $370-740 \mathrm{GBq} / \mu \mathrm{mol}$ and the radiochemical purities were greater than $98 \%$.

\section{Animal Preparation}

All animal procedures were performed in strict accordance with the National Institutes of Health Guide for the Care and Use of Laboratory Animals (18) and approved by Johns Hopkins University Animal Care and Use Committee. Male Wistar rats (250$350 \mathrm{~g}$ ) were housed in pairs, allowed food and water ad libitum, and studied. For PET, animals were anesthetized with $1.5 \%$ of isoflurane in oxygen and kept in the prone position on the scanner bed with the heart region centered in the gantry. The radiotracers were injected intravenously via the tail vein, and an aqueous solution of desipramine (DMI) hydrochloride was injected intraperitoneally before or after radiotracer injection, if applicable. Throughout the scanning procedure, body temperature was monitored by a rectal probe and maintained between $36.5^{\circ} \mathrm{C}$ and $37.5^{\circ} \mathrm{C}$ by a heating lamp. A single PET scan with 1 tracer under one of the subsequently described conditions was performed per animal, and after the PET scan the animals were euthanized with an overdose of isoflurane.

\section{Animal Groups}

Animals were assigned to 1 of 4 groups. In group 1 (control), each radiotracer-HED, PHEN, and EPI-was used in 4 animals without pharmacologic intervention. In group 2 (DMI chase), 4 animals per tracer were injected with radiotracer, and after $15 \mathrm{~min}$ into the PET scan, they received a 2-mg dose of DMI solution per kilogram intraperitoneally to inhibit reuptake of radiotracer by norepinephrine transporter (NET). In group 3 (DMI pretreatment), experiments were performed on a single rat per tracer. The animal received a 2-mg dose of DMI solution per kilogram intraperitoneally $15 \mathrm{~min}$ before injection of each of the 3 radiotracers to inhibit uptake of radiotracer by NET. In the fourth group, 4 animals were injected with low-specific-radioactivity EPI (9-18 $\mathrm{MBq} / \mu \mathrm{mol})$ without pharmacologic intervention.

\section{PET}

Imaging was performed using the Johns Hopkins University small-animal PET system (eXplore Vista; GE Healthcare). The characteristics of this scanner have been described previously (19). In brief, the system has a transaxial field of view (FOV) of $6.7 \mathrm{~cm}$ and an axial FOV of $4.8 \mathrm{~cm}$, achieving an in-plane spatial and volume resolution of approximately $1.4 \mathrm{~mm}$ and $2.9 \mathrm{~mm}^{3}$, respectively.

Under anesthesia, animals were placed in the prone position in the scanner gantry, and radiotracer $(18.5 \mathrm{MBq})$ was injected via the tail vein, 10-15 min after synthesis. Image acquisition and radiotracer injection started simultaneously, and a dynamic sequence (40 frames: $6 \times 5 \mathrm{~s}, 10 \times 15 \mathrm{~s}, 12 \times 30 \mathrm{~s}, 6 \times 60 \mathrm{~s}, 3 \times 300 \mathrm{~s}$, and $3 \times 600 \mathrm{~s}$ ) was acquired over $60 \mathrm{~min}$.

\section{Image Reconstruction and Time-Activity Curves}

Transaxial images were reconstructed by a 2 -dimensional iterative algorithm (ordered-subset expectation maximization, 1 iteration, and 16 subsets). Image data were not corrected for attenuation and scatter. For an organ-based analysis, heart and liver were identified on PET images, and regions of interest (ROIs) associated with the left ventricle (LV) chamber, LV lateral wall, LV septum, LV apex, and liver were drawn. ROIs initially indicate values in radioactivity per cubic centimeter. We used the injected amount of radioactivity to transform values to percentage injected dose per cubic centimeter. Then, assuming a relative density of $1 \mathrm{~g} / \mathrm{cm}^{3}$, multiplication with the true animal weight in grams was performed to obtain percentage of standardized uptake value (SUV). This was 
done to normalize for the differences in rat weight and administered dose and to allow for comparison of intergroup with interorgan data. In animal groups 1, 2, and 4, ROIs were drawn on the tomographic images on frame 40 (last frame). In group 3, because of absence of specific uptake in later frames, an earlier frame (frame 10) was selected. Decay-corrected time-activity curves were presented as percentage SUV; all percentage SUVs in the study presented a mean of measurements in 4 rats with each of the 3 radiotracers in groups 1 , 2 , and 4 . The percentage SUV for each radiotracer in group 3 was obtained from a single rat.

\section{Retention Index and Washout Rate}

For detailed analysis of myocardial kinetics, volumetric sampling was used to create polar maps of activity distribution throughout the left ventricular myocardium (20,21). A non-metabolite-corrected input function was derived from a circular ROI in the LV cavity. Then, a non-metabolite-corrected radiotracer retention index was determined by normalizing myocardial tracer activity from 30 to $40 \mathrm{~min}$ to the integral of the arterial input function. The clearance of radiotracers was defined from data between 10 and 60 min after injection that were fitted to a monoexponential function and expressed as \%/min. The average non-metabolite-corrected LV myocardial retention index and washout rate were computed from septal, lateral, anterior, inferior, and apical regions.

\section{Statistical Analysis}

Data are reported as mean $\pm \mathrm{SD}$, which was calculated by averaging all ROIs for all animals in a given study to include both subject and group variability for SD. Comparisons of 2 mean values were performed with an unpaired Student $t$ test. A $P$ value of less than 0.05 was considered significant.

\section{RESULTS}

The LV and liver were visualized clearly after HED, PHEN, and EPI injection in untreated control rats (Fig. 2). The contrast and quality of HED tomographic images were better than those for PHEN and EPI (radioactivity ratio of LV lateral wall to liver at $55 \mathrm{~min}$ was $0.96,0.44$, and 0.48 for HED, PHEN, and EPI, respectively). Tracer kinetics in heart and liver differed significantly among tracers, as described in the following sections.

\section{HED}

HED showed rapid uptake and sustained retention in the rat myocardium without medical intervention (Fig. 3A). Using the DMI chase protocol, initial retention was followed by rapid washout after DMI administration (Fig. 3B). After DMI pretreatment, rapid washout without retention in the myocardium was observed (Fig. 3C). The average nonmetabolite-corrected LV retention index at the end of the scan was $7.38 \% \pm 0.82 \% / \mathrm{min}$ and $3.28 \% \pm 0.60 \% / \mathrm{min}$ for the control group and DMI chase. In the single animal after DMI pretreatment, it was $0.21 \% \pm 0.35 \% / \mathrm{min}$ (Table 1 ). The LV retention index was lower in DMI chase $(P<0.05)$ than in untreated rats. The average $\mathrm{LV}$ washout rate of HED was significantly faster after DMI chase $(1.86 \% \pm 0.57 \% / \mathrm{min})$ than in controls $(0.13 \% \pm 0.23 \% / \mathrm{min})$ (Table 1$)$.

In the liver, HED displayed slow uptake in control rats, slow initial uptake followed by more rapid uptake after DMI chase, and rapid uptake immediately after radiotracer injection after DMI pretreatment. The PET-measured HED liver uptake values at $30 \mathrm{~s}$ and $55 \mathrm{~min}$ in controls, DMI chase, and DMI pretreated rats were $300 \%$ and $500 \%$ SUV, $300 \%$ and $600 \%$ SUV, and $600 \%$ and $800 \%$ SUV, respectively (Figs. 3A-C).

\section{PHEN}

PHEN showed rapid uptake followed by monoexponential washout in the myocardium of control rats (Fig. 4A). Myocardial kinetics of PHEN in controls and after DMI chase were mostly similar (Figs. 4A and 4B). DMI chase did not enhance washout of PHEN in the myocardium. In the single DMI pretreated rat, PHEN showed rapid washout without retention in the myocardium (Fig. 4C). The average nonmetabolite-corrected LV retention index of PHEN radioactivity at the end of the scan was $3.43 \% \pm 0.45 \% / \mathrm{min}$,

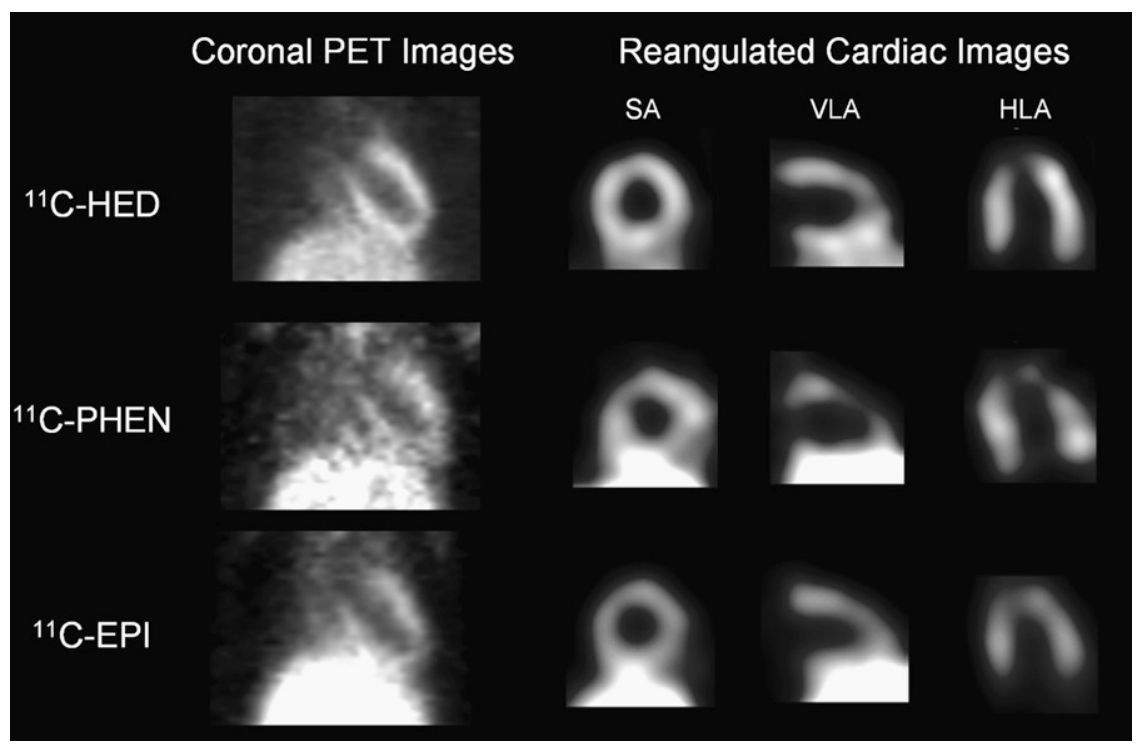

FIGURE 2. Representative static coronal PET images and reangulated, smoothed cardiac images of rats, acquired between 50 and $60 \mathrm{~min}$ after intravenous injection of HED, PHEN, or EPI. Shown on right are midventricular short-axis (SA), vertical long-axis (VLA), and horizontal long-axis slices (HLA). Liver is visualized inferior to left ventricle. 

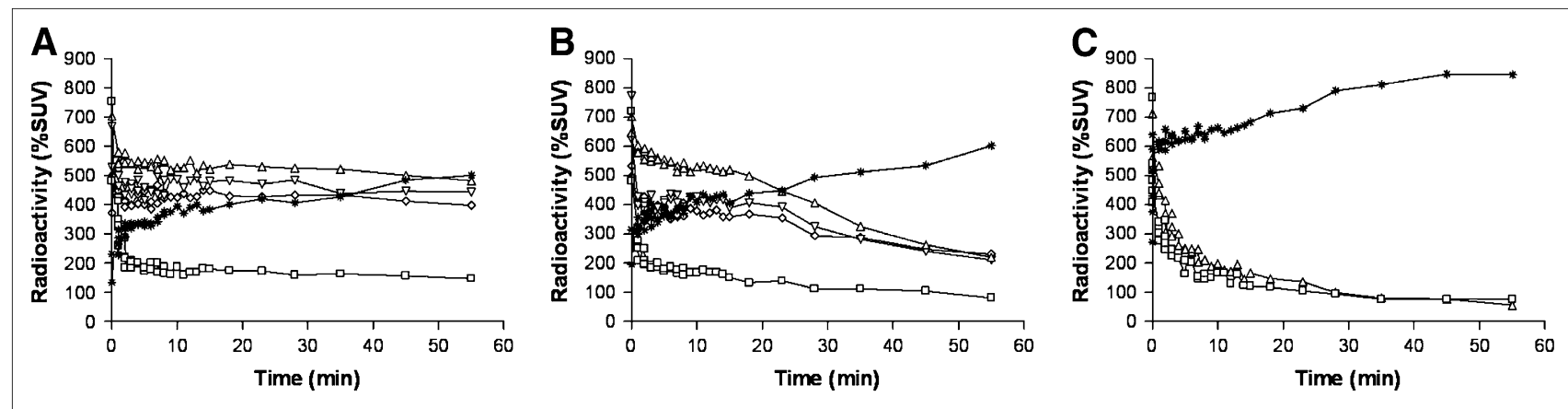

FIGURE 3. Myocardial time-activity curves for LV chamber $(\square)$, LV lateral wall $(\triangle)$, LV septum $(\nabla)$, LV apex $(\diamond)$, and liver $\left({ }^{*}\right)$ in rats after intravenous injection of HED, without pharmacologic intervention (A), with intraperitoneal administration of $2 \mathrm{mg}$ of DMl solution per kilogram 15 min after tracer injection (DMI chase) (B), and after intraperitoneal administration of 2 mg of DMl solution per kilogram 15 min before tracer injection (DMI block) (C). A and B show data averaged from 4 rats, and $C$ shows observation from 1 rat (LV septum and LV apex were included in FOV but not detectable in C; kinetics in anterior wall were also analyzed but did not differ from other myocardial regions and are therefore not shown).

$2.95 \% \pm 0.51 \% / \mathrm{min}$, and $0.24 \% \pm 0.25 \% / \mathrm{min}$, respectively, for control group, DMI chase group, and the single DMI pretreated rat (Table 1). The average LV washout rate of PHEN was not significantly faster in DMI chase rats $(1.51 \% \pm 0.16 \% / \mathrm{min})$ than in the control group $(1.13 \% \pm$ $0.35 \% / \mathrm{min}$ ) (Table 1).

In the liver, PHEN showed rapid uptake and subsequent washout between 0 and $15 \mathrm{~min}$ followed by sustained retention until $60 \mathrm{~min}$. The PET-measured PHEN liver uptake at $30 \mathrm{~s}$ and $55 \mathrm{~min}$ in the control group, DMI chase, and DMI pretreated rats were $600 \%$ and $450 \%$ SUV, $600 \%$ and $450 \%$ SUV, and $650 \%$ and $450 \%$ SUV, respectively (Figs. 4A-C).

\section{EPI}

EPI showed rapid uptake and subsequent biphasic washout from the myocardium with a first rapid phase followed by slower clearance and retention of radioactivity in the second phase (Fig. 5A). Lower specific radioactivity and DMI chase did not affect myocardial tracer kinetics significantly (Figs. $5 \mathrm{~B}$ and $5 \mathrm{C}$ ). Myocardial retention was not observed in the single DMI pretreated rat (Fig. 5D). The average nonmetabolite-corrected LV retention index at the end of the scan was $4.24 \% \pm 0.59 \% / \mathrm{min}, 3.87 \% \pm 0.81 \% / \mathrm{min}, 4.02 \% \pm$ $0.99 \% / \mathrm{min}$, and $0.22 \% \pm 0.30 \% / \mathrm{min}$, respectively, for the control group, low-specific-radioactivity group, DMI chase group, and the single DMI pretreated rat (Table 1). The average LV washout rate of EPI was not significantly faster in DMI chase rats $(0.50 \% \pm 0.24 \% / \mathrm{min})$ and the low-specificradioactivity group $(0.61 \% \pm 0.28 \% / \mathrm{min})$ than in the control group $(0.69 \% \pm 0.17 \% / \mathrm{min})$ (Table 1$)$.

In the liver, EPI showed rapid uptake, followed by sustained retention until $60 \mathrm{~min}$. The PET-measured EPI liver uptake at $30 \mathrm{~s}$ and $55 \mathrm{~min}$ in control, low-specificradioactivity, DMI chase, and DMI pretreated rats was $400 \%$ and $550 \%$ SUV, $500 \%$ and $700 \%$ SUV, $450 \%$ and $550 \%$ SUV, and $550 \%$ and $700 \%$ SUV, respectively (Figs. $5 \mathrm{~A}-5 \mathrm{D})$.

TABLE 1

Non-Metabolite-Corrected Myocardial Retention Index and Washout Rate of Radiotracers With and Without Treatment With DMI

\begin{tabular}{|c|c|c|c|c|c|c|}
\hline \multirow[b]{2}{*}{ Radiotracer } & \multicolumn{3}{|c|}{$\begin{array}{l}\text { Retention index } \\
\text { (\%/min at } 40 \mathrm{~min})\end{array}$} & \multicolumn{3}{|c|}{$\begin{array}{l}\text { Washout rate }(\% / \mathrm{min} \\
\text { between } 10 \text { and } 60 \mathrm{~min})\end{array}$} \\
\hline & $\begin{array}{l}\text { Without } \\
\text { intervention } \\
(n=4)\end{array}$ & $\begin{array}{c}\text { DMI } \\
\text { chase } \\
(n=4)\end{array}$ & $\begin{array}{c}\text { DMI } \\
\text { pretreatment } \\
(n=1)\end{array}$ & $\begin{array}{l}\text { Without } \\
\text { intervention } \\
(n=4)\end{array}$ & $\begin{array}{c}\text { DMl } \\
\text { chase } \\
(n=4)\end{array}$ & $\begin{array}{l}\text { DMI } \\
\text { pretreatment } \\
(n=1)\end{array}$ \\
\hline $\begin{array}{l}\text { HED } \\
\text { PHEN } \\
\text { EPI } \\
\text { EPI (low specific radioactivity) }\end{array}$ & $\begin{array}{l}7.38 \pm 0.82 \\
3.43 \pm 0.45 \\
4.24 \pm 0.59 \\
3.87 \pm 0.81\end{array}$ & $\begin{aligned} 3.28 & \pm 0.60^{*} \\
2.95 & \pm 0.51^{*} \\
4.02 & \pm 0.99 \\
& -\end{aligned}$ & $\begin{array}{l}0.21 \pm 0.35^{\dagger} \\
0.24 \pm 0.25^{\dagger} \\
0.22 \pm 0.30^{\dagger} \\
-\end{array}$ & $\begin{array}{l}0.13 \pm 0.23 \\
1.13 \pm 0.35 \\
0.50 \pm 0.24 \\
0.69 \pm 0.17\end{array}$ & $\begin{array}{l}1.86 \pm 0.57^{\star} \\
1.51 \pm 0.16 \\
0.61 \pm 0.28 \\
-\end{array}$ & $\begin{array}{l}\neq \\
\neq \\
\neq \\
-\end{array}$ \\
\hline $\begin{array}{l}{ }^{*} P<0.05 \text { vs. animals without } \\
{ }^{\dagger} \text { Single-case observation precl } \\
{ }^{\ddagger} \text { Complete washout of radiotra } \\
\text { Values are average } \pm \text { SD from }\end{array}$ & $\begin{array}{l}\text { rvention. } \\
\text { from myocar } \\
\text { ptal, lateral, in }\end{array}$ & $\begin{array}{l}\text { ting, but values } \\
\text { um in first } 10 \mathrm{n} \\
\text { erior, anterior, a }\end{array}$ & $\begin{array}{l}\text { markedly rec } \\
\text { in DMI pretrea } \\
\text { apex regions }\end{array}$ & $\begin{array}{l}\text { ced. } \\
\text { d rats. } \\
\text { left ventricle. }\end{array}$ & & \\
\hline
\end{tabular}



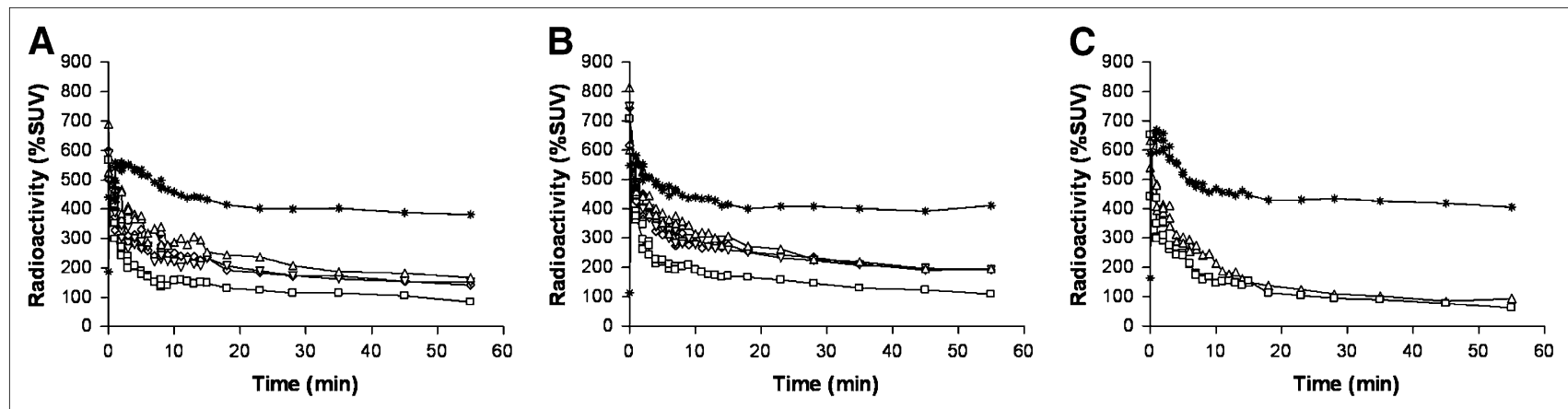

FIGURE 4. Myocardial time-activity curves of radioactivity in LV chamber $(\square)$, LV lateral wall $(\triangle)$, LV septum $(\nabla)$, LV apex $(\diamond)$, and liver $\left(^{*}\right)$ in rats after intravenous injection of PHEN, without pharmacologic intervention (A), with intraperitoneal administration of $2 \mathrm{mg}$ of DMI solution per kilogram 15 min after tracer injection (DMI chase) (B), and after intraperitoneal administration of 2 mg of DMI solution per kilogram 15 min before tracer injection (DMI block) (C). A and B show data averaged from 4 rats, and $C$ shows observation from 1 rat (LV septum and LV apex were included in FOV but not detectable in C; kinetics in anterior wall were also analyzed but did not differ from other myocardial regions and are therefore not shown).

\section{DISCUSSION}

Our dynamic in vivo PET studies of the sympathetic nervous system of the rat heart confirm the previously described kinetic properties of the catecholamine analog ${ }^{11} \mathrm{C}$-HED but showed differences in the handling of the physiologic catecholamine ${ }^{11} \mathrm{C}$-EPI and, to a lesser degree, of ${ }^{11} \mathrm{C}$-PHEN, characterized by high metabolic activity, high turnover, and clearance from the myocardium. This has important implications for the use of the rat heart as a model of cardiovascular disease, and it provides further insights into the biology of the rat sympathetic nervous system.

In general, the rat is an attractive species to study cardiovascular biology; compared with larger animals (pigs, dogs), they are easy to handle and reduce logistic complexity and costs. Rats are also preferred over smaller animals (mice), in which the small size of the heart still poses challenges for the robustness of noninvasive nuclear imaging. When compared with observations in humans (13-15) and isolated perfused rat hearts $(10,12,22)$, our multitracer characterization suggests that the cardiac sympathetic nervous system of the living rat is different and that conclusions from one species to the other must be interpreted with caution. For interpretation of our results, it is also important to emphasize that in vivo studies of sympathetic neuronal tracer kinetics may differ from studies of the isolated perfused heart because the peripheral nervous system and central nervous system are not preserved, and effects of systemic tracer degradation do not play a role in isolated perfused hearts.
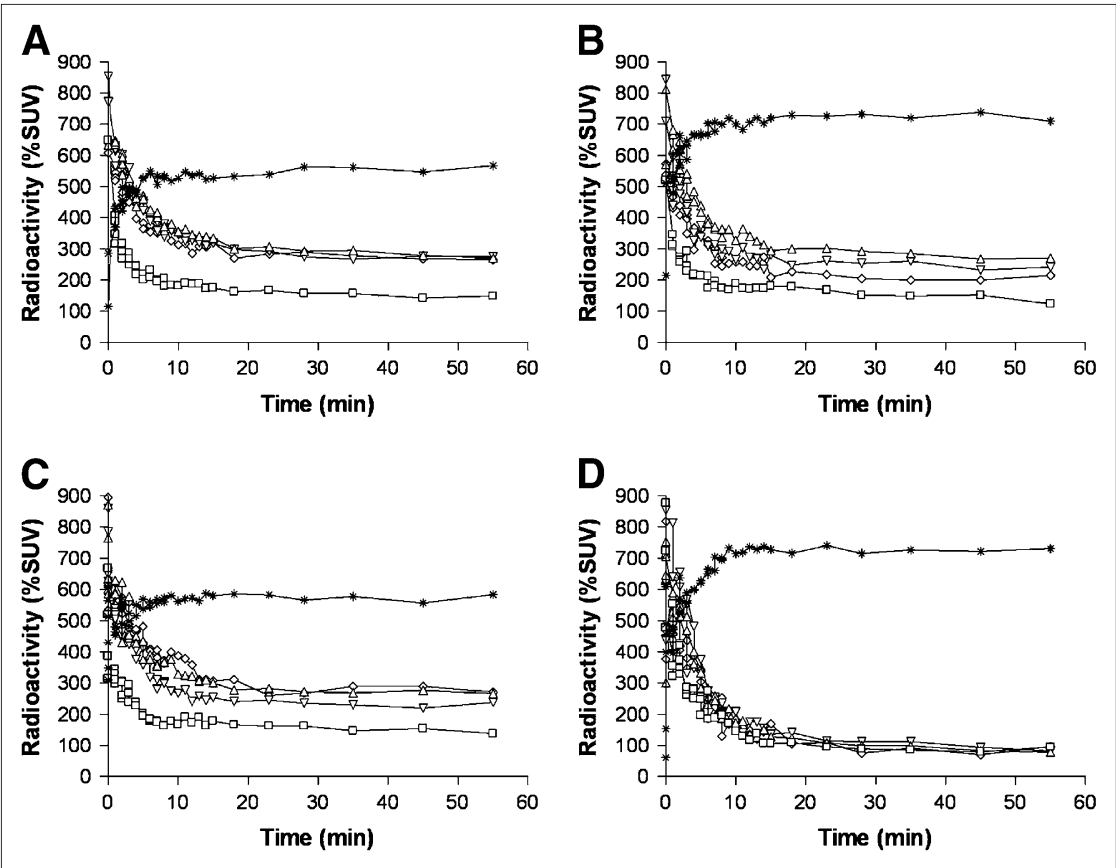

FIGURE 5. Myocardial time-activity curves of radioactivity in LV chamber $(\square)$, LV lateral wall $(\triangle)$, LV septum $(\nabla)$, LV apex $(\diamond)$, and liver $\left(^{*}\right)$ in rats after intravenous injection of $\mathrm{EPI}$, without pharmacologic intervention (A), with lower specific radioactivity without pharmacologic intervention (B), after intraperitoneal administration of $2 \mathrm{mg}$ of DMl solution per kilogram 15 min after tracer injection (DMI chase) (C), and after intraperitoneal administration of $2 \mathrm{mg}$ of DMl solution pre kilogram 15 min before tracer injection (DMI block) (D). A, B, and C show data averaged from 4 rats, and $D$ shows observation from 1 rat (LV septum and LV apex were included in FOV but not detectable in $\mathrm{D}$; kinetics in anterior wall were also analyzed but did not differ from other myocardial regions and are therefore not shown). 
Pretreatment with the specific NET blocker DMI was done in single animals in our study. In these observations, a significantly decreased radioactivity concentration of each of the 3 mentioned radiotracers in the myocardium was observed, a finding that is consistent with earlier, more extensive work $(10,12,22)$ and confirms their specific neuronal uptake via uptake-1. This supports the validity of these PET sympathoneuronal imaging agents for assessing myocardial sympathetic innervation.

For the MAO-resistant catecholamine analog HED, PETmeasured in vivo kinetics in the rat myocardium were comparable with isolated rat heart studies and cardiac PET in humans $(10,15,23,24)$. Avid uptake and subsequent stable retention was observed without DMI. The acceleration of myocardial clearance after DMI chase confirms earlier studies and suggests that HED is continuously taken up and released from nerve terminals. Vesicular storage does not seem to be a critical determinant of tracer retention, and myocardial retention seems to depend significantly on uptake-1-mediated reuptake into the nerve terminal. In the liver, accumulation of HED mainly reflects nonspecific uptake, which is accelerated and increased by DMI.

For the MAO-sensitive catecholamine analog PHEN, specific uptake was followed by myocardial washout as previously described. This washout has been attributed to leakage from intraneuronal vesicles and subsequent MAO metabolism, as confirmed by MAO blockade and comparison with the MAO-resistant analog D2-PHEN $(13,22)$. In humans, a mean clearance half-time of 55 min has been observed (13), whereas in untreated rats our clearance rates translate into a mean half-time of $44 \mathrm{~min}$, suggesting a somewhat more rapid metabolic degradation of PHEN due to either higher MAO activity or more pronounced vesicular leakage in rats than in humans. DMI chase did not significantly affect myocardial washout, suggesting that release and reuptake into the neuron are not significant contributors to PHEN kinetics. In the liver, PHEN-derived radioactivity shows an early peak with rapid clearance followed by stable retention that cannot be blocked by DMI. Early kinetics thus appear to reflect hepatic MAO metabolism, whereas sustained retention may be a combined effect of metabolite-derived activity, efflux from the liver, and influx from peripheral organs to the liver.

For the physiologic catecholamine EPI, kinetics differed from those previously described in humans and isolated perfused rat hearts, in which rapid initial uptake was followed by stable retention $(12,14)$. Our in vivo PET studies showed initial myocardial uptake followed by significant washout, suggesting a high myocardial turnover of catecholamines.

Several factors may contribute to this rapid turnover. For interpretation of these results, it is important to emphasize that EPI as a natural catecholamine is a substrate to all enzymatic metabolic pathways for catecholamines. The observation of stable retention in isolated perfused rat hearts and human hearts is explained by efficient storage of the tracer in neuronal vesicles, in which the tracer is protected from degradation after initial neuronal uptake via uptake-1/
NET $(12,14)$. The washout and lack of stable retention in the heart of living rats may theoretically be explained by intraneuronal vesicular leakage and subsequent metabolic degradation. The somewhat faster washout rates for PHEN (as a tracer of vesicular leakage) in rats than in humans may partially support this hypothesis. But the difference between species was subtle and not sufficient to explain the marked difference of EPI kinetics alone. Also, the lack of washout in isolated perfused rat hearts, in which axons are transsected and intraneuronal vesicular leakage may be even more pronounced, does not support this hypothesis. Second, nonneuronal degradation via the nonspecific uptake-2 and extraneuronal MAO may account for the washout of EPI. The very efficient blockade of myocardial EPI uptake by DMI pretreatment does not support this hypothesis, although very rapid extraneuronal degradation in the early phase, which may be difficult to separate from delivery and equilibrium effects, cannot be ruled out. Third, anesthesia may have influenced catecholamine metabolism and autonomic tone in rats and thereby explain the different results demonstrated in humans. Norepinephrine levels can rise during isoflurane anesthesia (25), but an increase in systemic norepinephrine should also affect tracer kinetics of HED, which was not observed in our study. Also, an isoflurane-induced increase in norepinephrine at the presynaptic site should reduce uptake of EPI, but EPI showed high initial uptake and then biphasic washout. Finally, the rapid washout of EPI radioactivity might be because of high neuronal activity and nerve firing, which is supported by the high heart rate found in rats. Rapid vesicular release from neurons may contribute to clearance from myocardium and subsequent extraneuronal metabolic degradation. We were not able to accelerate release of EPI by blockade of reuptake using a DMI chase, but the lack of influence of reuptake on the kinetics does not rule out vesicular release from neurons followed by rapid extraneuronal degradation.

We observed no difference in the EPI myocardial kinetic

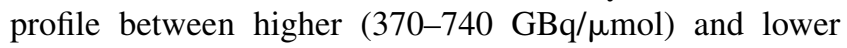
(9-18 $\mathrm{MBq} / \mu \mathrm{mol})$ specific radioactivity, suggesting that mass effects do not play a significant role in tracer kinetics $(26,27)$ and that neuronal uptake and storage are most likely high-capacity systems.

Although the myocardium was our primary target organ, we included the liver as a noncardiac organ in our analysis because it was in the FOV and showed high uptake that may interfere with cardiac image quality. Liver uptake was not blocked but rather increased by DMI, supporting the role of the liver as an organ of metabolic degradation in which radioactivity is mostly determined by nonspecific uptake of metabolites. Metabolism of catecholamines and its degradation products in hepatocytes, and the relative contribution of specific neuronal versus nonspecific nonneuronal uptake to liver kinetics, is not well understood but may be a subject of future dedicated studies.

Some limitations of our study need to be emphasized. First, we described kinetics of our tracers by simple measures 
of uptake and washout. Compartmental modeling of these tracers is not well established, and it is further complicated by inaccuracies of noninvasive definitions of the arterial input function in small-animal studies. Validation of kinetic modeling was thus considered beyond the scope of this project. Second, we were not able to measure plasma metabolites and obtain metabolite-corrected retention indices. Repeated blood sampling is difficult to perform in rodents because of the low total blood volume. Systemic tracer degradation may thus have contributed to inaccuracies of our retention measures. But the overall kinetic properties observed in our studies are unlikely to be a consequence of systemic effects only, and metabolite-independent measures such as the SUV are available for additional interpretation. Finally, we did not conduct our studies with multiple tracers in the same animals because we wanted to avoid residual effects of DMI or tracerderived radioactivity from prior imaging on a subsequent study. Intersubject differences thus may have contributed to higher variability between groups, but our results remain conclusive despite small sample sizes.

\section{CONCLUSION}

Our multitracer PET study suggests high turnover of physiologic catecholamines in the heart of living rats. Although the underlying molecular events are not fully elucidated, this may be because of rapid vesicular release and subsequent metabolic degradation. This observation has important implications for imaging studies of the cardiac sympathetic nervous system. First, the rat heart has limitations as a translational model of cardiac neuronal disease due to different pharmacokinetics of catecholamines. Second, conclusions from autonomic innervation studies in isolated perfused rat heart studies, in which this high turnover was not observed, to the in vivo environment may also be difficult because of lack of systemic and central nervous effects. And third, a single catecholaminergic tracer may not necessarily reflect the complete biology of sympathetic nerve terminals. The false neurotransmitter HED, for example, yielded highquality myocardial images in this study and comparable kinetics to humans but would not have allowed for identification of the turnover kinetics of true catecholamines such as epinephrine.

\section{ACKNOWLEDGMENTS}

We gratefully acknowledge Stephan Nekolla for providing MunichHeart/NM software for image analysis. We thank Riikka Lautamaki, Jurgen Seidel, and David M. Raffel for useful input to data analysis and interpretation.

\section{REFERENCES}

1. Levy MN. Sympathetic-parasympathetic interactions in the heart In: Kulbertus HE, Franck G, eds. Neurocardiology. New York, NY: Futura; 1988:85-98.
2. Rang HP, Dale MM, Ritter JM. Chemical mediators and the autonomic nervous system. Pharmacology. 4th ed. Edinburgh, U.K.: Churchill Livingstone; 1999:94-109.

3. Chierchia S, Davies G, Berkenboom G, et al. $\alpha$-Adrenergic receptors and coronary spasm: an elusive link. Circulation. 1984;69:8-14.

4. Watkins PJ, Mackay JD. Cardiac denervation in diabetic neuropathy. Ann Intern Med. 1980;92:304-307.

5. Corr PB, Gillis RA. Autonomic neural influences on the dysrhythmias resulting from myocardial infarction. Circ Res. 1978;43:1-9.

6. Lown B, Verrier RL, Rabinowitz SH. Neural and psychologic mechanisms and the problem of sudden cardiac death. Am J Cardiol. 1977;39:890-902.

7. Lown B, Verrier RL. Neural activity and ventricular fibrillation. $N$ Engl J Med. 1976;294:1165-1170.

8. Tipre DN, Goldstein DS. Cardiac and extracardiac sympathetic denervation in Parkinson's disease with orthostatic hypotension and in pure autonomic failure. J Nucl Med. 2005;46:1775-1781.

9. Bengel FM, Schwaiger M. Assessment of cardiac sympathetic neuronal function using PET imaging. J Nucl Cardiol. 2004;11:603-616.

10. DeGrado TR, Hutchins GD, Toorongian SA, et al. Myocardial kinetics of carbon-11-meta-hydroxyephedrine: retention mechanisms and effects of norepinephrine. J Nucl Med. 1993;34:1287-1293.

11. Del Rosario RB, Jung YW, Caraher J, et al. Synthesis and preliminary evaluation of $\left[{ }^{11} \mathrm{C}\right]-(-)$-phenylephrine as a functional heart neuronal PET agent. Nucl Med Biol. 1996;23:611-616.

12. Nguyen NT, DeGrado TR, Chakraborty P, et al. Myocardial kinetics of carbon11-epinephrine in the isolated working rat heart. J Nucl Med. 1997;38:780-785.

13. Raffel DM, Corbett JR, del Rosario RB, et al. Sensitivity of $\left[{ }^{11} \mathrm{C}\right]$ phenylephrine kinetics to monoamine oxidase activity in normal human heart. J Nucl Med. 1999;40:232-238.

14. Munch G, Nguyen NT, Nekolla S, et al. Evaluation of sympathetic nerve terminals with $\left[{ }^{11} \mathrm{C}\right]$ epinephrine and $\left[{ }^{11} \mathrm{C}\right]$ hydroxyephedrine and positron emission tomography. Circulation. 2000;101:516-523.

15. Schwaiger M, Kalff V, Rosenspire K, et al. Noninvasive evaluation of sympathetic nervous system in human heart by positron emission tomography. Circulation. 1990;82:457-464.

16. Chakraborty PK, Gildersleeve DL, Jewett DM, et al. High yield synthesis of high specific activity R-(-)-[ $\left.{ }^{11} \mathrm{C}\right]$ epinephrine for routine PET studies in humans. Nucl Med Biol. 1993;20:939-944.

17. Rosenspire KC, Haka MS, Van Dort ME, et al. Synthesis and preliminary evaluation of carbon-11-meta-hydroxyephedrine: a false transmitter agent for heart neuronal imaging. J Nucl Med. 1990;31:1328-1334.

18. Guide for the Care and Use of Laboratory Animals. Washington, DC: National Academy Press; 1996.

19. Wang Y, Seidel J, Tsui BM, et al. Performance evaluation of the GE Healthcare eXplore VISTA dual-ring small-animal PET scanner. J Nucl Med. 2006;47: 1891-1900.

20. Nekolla SG, Miethaner C, Nguyen N, et al. Reproducibility of polar map generation and assessment of defect severity and extent assessment in myocardial perfusion imaging using positron emission tomography. Eur J Nucl Med. 1998;25:1313-1321.

21. Hartmann F, Ziegler S, Nekolla S, et al. Regional patterns of myocardial sympathetic denervation in dilated cardiomyopathy: an analysis using carbon-11 hydroxyephedrine and positron emission tomography. Heart. 1999;81:262-270.

22. Raffel DM, Wieland DM. Influence of vesicular storage and monoamine oxidase activity on $\left[{ }^{11} \mathrm{C}\right]$ phenylephrine kinetics: studies in isolated rat heart. $\mathrm{J} \mathrm{Nucl} \mathrm{Med}$. 1999;40:323-330.

23. Malizia AL, Melichar JK, Rhodes CG, et al. Desipramine binding to noradrenaline reuptake sites in cardiac sympathetic neurons in man in vivo. Eur J Pharmacol. 2000;391:263-267.

24. Schwaiger M, Hutchins GD, Kalff V, et al. Evidence for regional catecholamine uptake and storage sites in the transplanted human heart by positron emission tomography. J Clin Invest. 1991;87:1681-1690.

25. Kushikata T, Hirota K, Kotani N, Yoshida H, Kudo M, Matsuki A. Isoflurane increases norepinephrine release in the rat preoptic area and the posterior hypothalamus in vivo and in vitro: relevance to thermoregulation during anesthesia. Neuroscience. 2005;131:79-86.

26. Fujita M, Zoghbi SS, Crescenzo MS, et al. Quantification of brain phosphodiesterase 4 in rat with (R)-[ $\left.{ }^{11} \mathrm{C}\right]$ Rolipram-PET. NeuroImage. 2005;26: 1201-1210.

27. Jagoda EM, Vaquero JJ, Seidel J, et al. Experiment assessment of mass effects in the rat: implications for small animal PET imaging. Nucl Med Biol. 2004;31: 771-779. 